\title{
An exploration of immunohistochemistry- based prognostic markers in patients undergoing curative resections for colon cancer
}

\author{
Astrid Louise Bjørn Bennedsen ${ }^{1 *}$, Luyi Cai ${ }^{2}$, Rune Petring Hasselager ${ }^{1}$, Aysun Avci Özcan', \\ Khadra Bashir Mohamed ${ }^{1}$, Jens Ole Eriksen³ ${ }^{3}$ Susanne Eiholm³ ${ }^{3}$ Michael Bzorek³ Anne-Marie Kanstrup Fiehn 1,3,4, \\ Thomas Vauvert F. Hviid ${ }^{4,5}$ and Ismail Gögenur ${ }^{1,4}$
}

\begin{abstract}
Background: The immune system recognizes and destroys cancer cells. However, cancer cells develop mechanisms to avoid detection by expressing cell surface proteins. Specific tumour cell surface proteins (e.g. HLA-G, PD-L1, CDX2) either alone or in combination with the relative presence of immune cells (CD3 and CD8 positive T-cells) in the tumour tissue may describe the cancer cells' ability to escape eradication by the immune system. The aim was to investigate the prognostic value of immunohistochemical markers in patients with colon cancer.
\end{abstract}

Methods: We conducted a retrospective study including patients diagnosed with pT3 and pT4 colon cancers. Immunohistochemical staining with HLA-G, PD-L1, CDX2, CD3, and CD8 was performed on tissue samples with representation of the invasive margin. PD-L1 expression in tumour cells and immune cells was reported conjointly. The expression of CD3 and CD8 was reported as a merged score based on the expression of both markers in the invasive margin and the tumour centre. Subsequently, a combined marker score was established based on all of the markers. Each marker added one point to the score when unfavourable immunohistochemical features was present, and the score was categorized as low, intermediate or high depending on the number of unfavourable stains. Hazard ratios for recurrence, disease-free survival and mortality were calculated.

Results: We included 188 patients undergoing colon cancer resections in 2011-2012. The median follow-up was 41.7 months, during which 41 (21.8\%) patients had recurrence and 74 (39.4\%) died. In multivariable regression analysis positive HLA-G expression ( $\mathrm{HR}=3.37,95 \% \mathrm{Cl}[1.64-6.93])$ was associated with higher recurrence rates, while a preserved $\mathrm{CDX} 2$ expression $(\mathrm{HR}=0.23,95 \% \mathrm{Cl}[0.06-0.85])$ was associated with a lower risk of recurrence. An intermediate or high combined marker score was associated with increased recurrence rates $(\mathrm{HR}=20.53,95 \% \mathrm{Cl}$ [2.68-157.32] and $\mathrm{HR}=7.56,95 \% \mathrm{Cl}$ [1.06-54.16], respectively). Neither high expression of PD-L1 nor high CD3-CD8 score was significantly associated with recurrence rates. Patients with a high CD3-CD8 score had a significantly longer DFS and OS.

Conclusions: In tumour cells, expression of HLA-G and loss of CDX2 expression were associated with cancer recurrence. In addition, a combination of certain tumour tissue biomarkers was associated with colorectal cancer recurrence.

\footnotetext{
${ }^{*}$ Correspondence: aslb@regionsjaelland.dk

${ }^{1}$ Center For Surgical Science (CSS), Department of Surgery, Zealand

University Hospital, Lykkebækvej 1,4600 Køge, Denmark

Full list of author information is available at the end of the article
} 
Keywords: Colon cancer, HLA-G, PD-L1, CDX2, CD3, CD8, Time to recurrence, Disease-free survival, Overall survival, Immunohistochemistry

\section{Background}

Immune evasion was presented as an emerging hallmark of cancer in 2011 [1]. In the tumour microenvironment, immune cells interact continuously with the cancer cells during tumorigenesis, a process that takes several years $[2,3]$. Through T-cell activation the adaptive immune system has the capacity to impair tumorigenesis, when tumour-associated antigens are presented [4]. However, the cancer cells often escape immune surveillance by activation of immune checkpoint pathways, thus avoiding anticancer immunity [5]. In recent years, immune checkpoint inhibitors have been introduced [6].

As clinical outcome varies substantially among patients diagnosed within the same tumour stage this emphasizes the need for further refinement of the current classification [7]. The Immunoscore $\odot$, which is based on the expression of cluster of differentiation 3 (CD3) and CD8 on tumour-infiltrating lymphocytes (TILs) in the tumour centre and in the invasive margin, has shown superiority as a prognostic marker over Union for International Cancer Control (UICC)-TNM classification and highlighted the importance of TILs and anti-cancer immunity $[7,8]$.

Several other immunohistochemical (IHC) markers are under investigation as promising prognostic or predictive biomarkers. Human leukocyte antigen G (HLA-G) is a non-classical human leukocyte antigen (HLA) class Ib molecule that has immune modulatory properties [9]. The expression of HLA-G is found in both physiological and pathological conditions [10]. HLA-G can impair the function of T-cells, B-cells, and natural killer (NK) cells through several inhibitory pathways, and is a marker of immune evasion [11-13]. Recently, HLA-G expression has been associated with a worsened prognosis in patients with colorectal cancer [14-17].

The programmed death 1 (PD-1) pathway is involved in inhibition of the immune response and the exhaustion of T-cells [18]. Programmed death-ligand 1 (PDL1) is expressed constitutively on T-cells, B-cells, macrophages and other hematopoietic and non-hematopoietic cells, and is inducible through cytokines and in-trans binding of the immune checkpoint PD-1 [19]. Cancer cells can express PD-L1, and several published studies have investigated the role of PD-L1 both as a prognostic marker and a predictive marker for immune checkpoint blockade [6, 20-24].

Homeobox protein CDX2 (CDX2) is a marker of differentiation of colon cancer cells and has been proposed as a strong prognostic marker in patients with colon cancer [25].

The aim of this study was to explore the expression patterns of HLA-G, PD-L1, and CDX2 as well as CD3 and CD8 in a cohort of patients diagnosed with pT3 and pT4 colon cancers, and to investigate their value as prognostic markers individually and in a combined model.

\section{Materials and methods \\ Patients}

We conducted a retrospective study on archived tissue samples. The study was reported in accordance with the REMARK checklist [26]. Consecutive patients, who underwent colon cancer resection and were diagnosed with pT3 and pT4 tumours at Zealand University Hospital from 1st January 2011 until 31st December 2012, were included in the study. In the diagnostic routine setting a standardized pathological examination of the specimens had been performed according to national guidelines at the time of diagnosis. Briefly, at the macroscopic examination representative areas demonstrating key tumour features were identified and selected for paraffin embedding. Histopathological examination and tumour staging were performed according to the UICC-TNM classification. All histologic diagnoses are coded according to the Systematized Nomenclature of Medicine. Patients were searched from the records using the codes adenocarcinoma and resection combined with either $p T 3$ or $p T 4$. Exclusion criteria were patients that were under 18 years, had a history of previous cancer, had insufficient amount of tumour tissue for the supplementary IHC stainings, were registered in the Danish Registry for Use of Tissue (refusing to have their tissue used in research), had a preoperative stent, or who had received preoperative chemotherapy or radiotherapy.

\section{Tissue samples}

Haematoxylin and eosin (H\&E) stained slides from each patient were retrieved from the archive of the Department of Pathology, Zealand University Hospital, and reviewed by a consultant Pathologist. For each patient, one slide with representation of the invasive margin was selected, and the corresponding formalin-fixed paraffinembedded (FFPE) block was retrieved for IHC stainings.

\section{Immunohistochemical stainings}

Sections with a thickness of $4 \mu \mathrm{m}$ were cut and slides were deparaffinised and rehydrated. Immunohistochemical 
stainings were performed using anti-HLA-G clone $4 \mathrm{H} 84$ (Exbio, Praha, Czech Republic, cat.no 11-499-C100), anti-PD-L1 clone 22C3 (Agilent/Dako, Glostrup, Denmark, cat.no M3653), anti-CDX2 clone DAK-CDX2 (Agilent/Dako, cat. no. GA080), anti-CD8 clone C8/144B (Agilent/Dako, cat. no. GA623) and anti-CD3 clone LN10 (Leica/Triolab AS, Broendby, Denmark, cat. no. NCL-LCD3-565). All stainings was performed on the automated instrument Omnis (Agilent/Dako). For PD-L1, the protocol has been described in detail elsewhere [27]. Briefly, and for all other markers, antigen retrieval was accomplished using EnVision ${ }^{\mathrm{TM}}$ FLEX Target Retrieval Solution, High pH (Agilent/Dako, cat.no GV804) for $24 \mathrm{~min}$ at $97^{\circ} \mathrm{C}$. After pre-treatment, slides were incubated with the primary antibodies HLA-G (1:600), CDX2 (ReadyTo-Use/RTU), CD8 (RTU) and CD3 (1:50) for $30 \mathrm{~min}$ at $32^{\circ} \mathrm{C}$. The reactions were detected using the standard polymer technique EnVision ${ }^{\mathrm{TM}}$ FLEX /HRP Detection Reagent (Agilent/Dako, cat. no GV800), signal intensity was enhanced using EnVision ${ }^{\mathrm{TM}}$ FLEX+ Mouse (LINKER) (Agilent/Dako, cat. no GV821) and visualized using EnVision $^{\text {TM }}$ Flex DAB + Chromogen system (Agilent/Dako, cat. no. GV825) following the instructions given by the manufacturer. Finally, sections were counterstained with Haematoxylin and mounted with pertex.

\section{Evaluation of immunohistochemical stainings}

HLA-G and CDX2 were assessed manually and semiquantitatively. All slides were evaluated by two assessors blinded to all clinical data. At least one was a gastrointestinal pathologist. We reported HLA-G expression as either negative $(<10$ positive cells) or positive $(\geq 10$ positive cells per whole slide). A positive cell was defined as cytoplasmic or membrane staining of any intensity. CDX2 expression was classified as preserved (strong positive nuclear staining in $>75 \%$ tumour cells) or reduced $(<75 \%$ tumour cells).

PD-L1, CD3 and CD8 stained tissue slides were assessed digitally and classified as high or low based on the median value of our dataset. Slides were digitized at 20x using a Leica SCN400 slide scanner (Leica Biosystems, Nussloch Germany). Algorithms for PD-L1, CD3 and CD8 stainings were developed in the TissueIA software part of Digital Image Hub (version 4.0.5) (Leica Biosystems, Nussloch Germany). The algorithms detected all intact cell nuclei based on haematoxylin counterstaining and the brown membrane DAB staining. The algorithms were adjusted and fine-tuned in close collaboration with a pathologist comparing the digital reads with manual counting until sufficient compliance was obtained.

PD-L1 was analysed as a combined positive score with percentage of all positive cells (tumour cells, lymphocytes and macrophages) divided by the total number of cells. Membrane staining in at least $75 \%$ of the membrane area were required for a cell to be classified as positive. Necrotic areas and areas of healthy tissue were excluded manually on all slides.

CD3 and CD8 expression was reported as percentages of all positive cells divided by total number of cells in the invasive margin and in the tumour centre, respectively. The invasive margin and the tumour centre was identified and delineated manually on each slide. A positive cell was defined as strong cytoplasmic staining with membranous accentuation. The median value of the percentages of CD3 and CD8 positive cells in the invasive margin and in the central tumour, respectively, was used as cut-off yielding a score of either 0 or 1 . Tumours with a score of 1 for both $\mathrm{CD} 3$ and $\mathrm{CD} 8$ in the two compartments were classified as high CD3-CD8 infiltration, while tumours with any score of 0 was classified as low CD3-CD8 infiltration.

Finally, we computed a combined marker score based on features of the markers that were expected as related to immune escape by tumours. Each marker was an addend in the score with a value of zero (favourable) or one (unfavourable) depending on the expression pattern. The following unfavourable expression patterns each added one point to the score: positive HLA-G expression, low PD-L1 expression, reduced CDX2 expression, and low CD3-CD8 immune cell infiltration. The points were summarized and patients with score 0 had a low combined marker score, patients with score 1-2 had an intermediate combined marker score, and patients with score 3-4 had a high combined marker score. Patients with a low combined marker score were expected to have a favourable prognosis, while patients with a high combined marker score were expected to have an unfavourable prognosis.

Figure 1 shows representative positive and negative IHC stains of all markers.

\section{Data collection and variables}

Patient data were collected retrospectively from patient files. Baseline data consisted of age at surgery, sex, American Society of Anaesthesiologists (ASA) physical status grade, smoking status, location of primary tumour, preoperative metastases, surgery type, primary surgical procedure, 30 days postoperative complications graded by the Clavien-Dindo classification, perioperative blood transfusions, UICC stage, histological subtype, microscopic assessment of the resection margin, and information on postoperative chemotherapy. Microsatellite status, defined as either microsatellite instable (MSI) or microsatellite stabile (MSS), was collected from pathology reports, and was based on IHC for mismatch repair proteins (expression of MLH1 and MSH2, eventually 


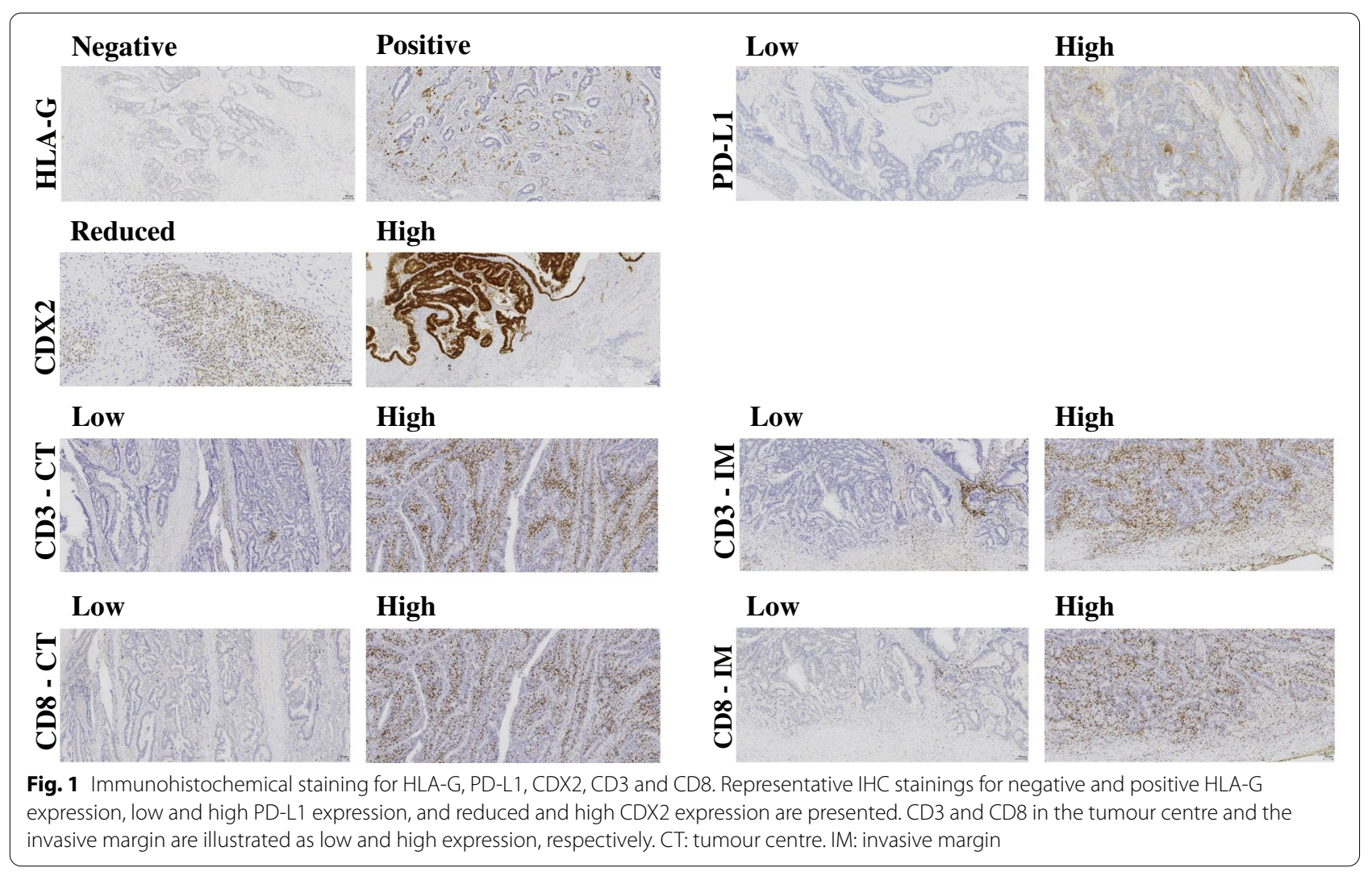

combined with expression of MSH6 and PMS2 for patients with resections performed in 2012).

The primary outcome was time to recurrence defined as time in months from surgery until recurrence was recorded. Recurrence events were defined as any recorded event of clinical recurrence in the patient files. Secondary outcomes were overall survival (OS) and disease-free survival (DFS) defined as time until death or time to either recurrence or death, respectively. The end of the follow-up period was December 2017. Patients were censored at the last postoperative control for time to recurrence and DFS analyses. The patient files were linked to the Danish Central Person Registry, which ensures complete follow-up for mortality analyses.

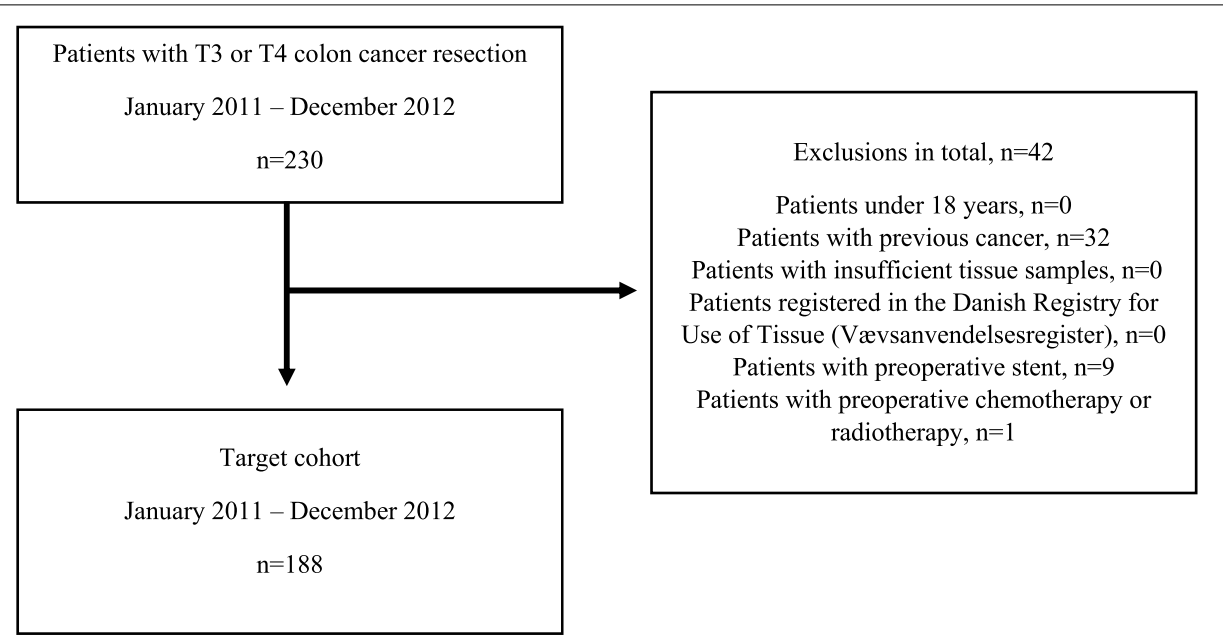

Fig. 2 Cohort definition. A total of 188 patients were included in this study after excluding 42 patients due to the exclusion criteria 
Table 1 Baseline Characteristics. The total cohort of 188 patients with UICC stage II-IV colon cancer

\begin{tabular}{|c|c|c|}
\hline & n (\%) & Missing, \% \\
\hline N & 188 & \\
\hline Age, years (median, IQR) & $71.50[65.00,79.00]$ & 0.0 \\
\hline \multicolumn{3}{|l|}{ Sex } \\
\hline Female & $99(52.7)$ & \multirow[t]{2}{*}{0.0} \\
\hline Male & $89(47.3)$ & \\
\hline \multicolumn{3}{|l|}{ ASA Score } \\
\hline 1 & $17(11.6)$ & \multirow[t]{4}{*}{22.3} \\
\hline$\|$ & $105(71.9)$ & \\
\hline III & $24(16.4)$ & \\
\hline IV & $0(0.0)$ & \\
\hline \multicolumn{3}{|l|}{ Tobacco } \\
\hline Current smoker & $33(17.6)$ & \multirow[t]{2}{*}{0.5} \\
\hline Former or never smoker & $154(82.4)$ & \\
\hline \multicolumn{3}{|l|}{ Tumour localization } \\
\hline Right-sided & $103(54.8)$ & \multirow[t]{2}{*}{0.0} \\
\hline Left-sided & $85(45.2)$ & \\
\hline \multicolumn{3}{|l|}{ Preoperative liver metastases } \\
\hline No & $174(92.6)$ & \multirow[t]{2}{*}{0.0} \\
\hline Yes & $14(7.4)$ & \\
\hline \multicolumn{3}{|l|}{ Preoperative lung metastases } \\
\hline No & $182(96.8)$ & \multirow[t]{2}{*}{0.0} \\
\hline Yes & $6(3.2)$ & \\
\hline \multicolumn{3}{|l|}{ Urgency } \\
\hline Elective & $157(83.5)$ & \multirow[t]{2}{*}{0.0} \\
\hline Acute & $31(16.5)$ & \\
\hline \multicolumn{3}{|l|}{ Procedure type } \\
\hline Right hemicolectomy including transverse resection & $104(55.3)$ & \multirow[t]{3}{*}{0.0} \\
\hline Left hemicolectomy & $76(40.4)$ & \\
\hline Colectomy & $8(4.3)$ & \\
\hline \multicolumn{3}{|l|}{ Perioperative blood transfusion } \\
\hline No & $162(86.6)$ & \multirow[t]{2}{*}{0.5} \\
\hline Yes & $25(13.4)$ & \\
\hline \multicolumn{3}{|l|}{ Postoperative complications (Clavien-Dindo) } \\
\hline 0 (no complications) & $126(67.4)$ & \multirow[t]{4}{*}{0.5} \\
\hline $1-2$ & $13(7.0)$ & \\
\hline $3-4$ & $40(21.4)$ & \\
\hline 5 (death) & $8(4.3)$ & \\
\hline \multicolumn{3}{|l|}{ UICC stage } \\
\hline$\|$ & $90(47.9)$ & \multirow[t]{3}{*}{0.0} \\
\hline III & $82(43.6)$ & \\
\hline IV & $16(8.5)$ & \\
\hline \multicolumn{3}{|l|}{ Histological type } \\
\hline Adenocarcinoma NOS, high or moderate differentiated & $124(66.0)$ & 0.0 \\
\hline Adenocarcinoma, poorly differentiated & $32(17.0)$ & \\
\hline Mucinous adenocarcinoma & $29(15.4)$ & \\
\hline Signet ring cell carcinoma & $2(1.1)$ & \\
\hline Other carcinoma type & $1(0.5)$ & \\
\hline Microsatellite status & & \\
\hline MSS & $144(76.6)$ & 0.0 \\
\hline
\end{tabular}


Table 1 (continued)

\begin{tabular}{lll}
\hline & $\mathbf{n}(\%)$ & Missing, \% \\
\hline MSI & $44(23.4)$ & \\
Resection margin & $170(94.4)$ & 4.3 \\
R0 (no residual tumor cells) & $10(5.6)$ \\
R1 (micro- or macroscopic residual tumor) & \\
Adjuvant chemotherapy & $107(56.9)$ \\
$\quad$ No & $81(43.1)$ \\
Yes & $41.72[10.56,59.82]$ & 0.0 \\
Follow-up time, months (median, lQR) & 0.0 \\
\hline
\end{tabular}

Absolute numbers with percentages in parentheses unless stated otherwise

IQR inter-quartile range, ASA American Society of Anesthesiologists Physical Status Score, UICC Union for International Cancer Control Score, NOS not otherwise specified, MSS microsatellite stability, MSI microsatellite instability

\section{Statistical analysis methods}

For baseline characteristics, the categorical variables were reported as number of patients and frequencies and the continuous variables as medians with inter-quartile ranges (IQR). Patients were classified according to expression of IHC markers and compared using MannWhitney $\mathrm{U}$ test for continuous variables and chi-squared test for categorical variables.

Time-to-event data were visualized using AalenJohansen estimates for cumulative incidence plots for recurrence and Kaplan-Meier plots for DFS and OS. Groups were compared using log-rank test for KaplanMeier estimates and Gray's test for cumulative incidence, thereby accounting for mortality as a competing risk for cancer recurrence [28].

Based on existing literature and knowledge, we selected the following variables as the most important potential confounders: ( $<70$ or $\geq 70$ years $)$, microsatellite status (MSS or MSI), UICC stage (II, III or IV) and sidedness of tumour (right-sided or left-sided). We used multivariable Cox regression to adjust for the confounders and assessed the association of each biomarker with the outcomes separately. The variables overall met the proportional hazards assumption which was assessed by plots of Schoenfeld residuals. To account for mortality as a competing risk for recurrence, we applied the subdistribution hazards approach by Fine and Gray for these analyses [29]. Estimates are presented as hazard ratios (HR) with 95\% confidence intervals (CI).

For all tests, $p$-values below 0.05 were considered statistically significant. We performed the statistical analyses using $R$ version 3.6.1. ( $R$ Core Team (2019). R: A language and environment for statistical computing. $R$ Foundation for Statistical Computing, Vienna, Austria. URL https://www.R-project.org/).

\section{Results}

\section{Participant characteristics}

A total of 188 patients with pT3 and pT4 colon cancer tumours were included (Fig. 2). The median age at surgery was $71.5(65-79)$ years, and $99(52.7 \%)$ of the patients were females. The tumours were primarily rightsided $(n=103,(54.8 \%))$ and $44(23.4 \%)$ of the tumours were MSI. Ninety (47.9\%) patients were UICC stage II, 82 (43.6\%) were stage III and 16 (8.5\%) stage IV. The median follow-up after resection was 41.7 (10.6-59.8) months (Table 1). During follow-up 41 (21.8\%) patients experienced recurrence and 74 (39.4\%) died. Eight patients did not participate in the postoperative follow-up programme, and were censored from the last outpatient visit when registering recurrence status.

\section{HLA-G expression status}

A total of 17 (9.0\%) patients were classified as HLA-Gpositive (Table 2). The HLA-G-positive cancer cells were primarily located in the invasive margin or in the deeper compartments of the tumour (data not shown).

Of the HLA-G-positive patients, eight (47.1\%) experienced cancer recurrence and 11 (64.7\%) died. In the HLA-G-negative group, the death and recurrence numbers were 63 (36.8\%) and 33 (19.3\%), respectively. In the unadjusted non-parametric analysis there was significant difference between the groups for recurrence $(p=0.003$, Fig. 3), DFS ( $p=0.001$, Fig. 4$)$ and OS ( $p=0.035$, Fig. 5). Confounder adjusted multivariable regression analyses yielded higher recurrence rates, HR 3.37 (95\%CI [1.64-6.93], Fig. 6), and worse DFS, $\mathrm{HR}=2.28$ (95\%CI [1.24-4.18], Fig. 7), for HLA-G-positive individuals. The regression analysis for $\mathrm{OS}$ was not significant $(\mathrm{HR}=1.65$, 95\%CI [0.86-3.15], Fig. 8). 


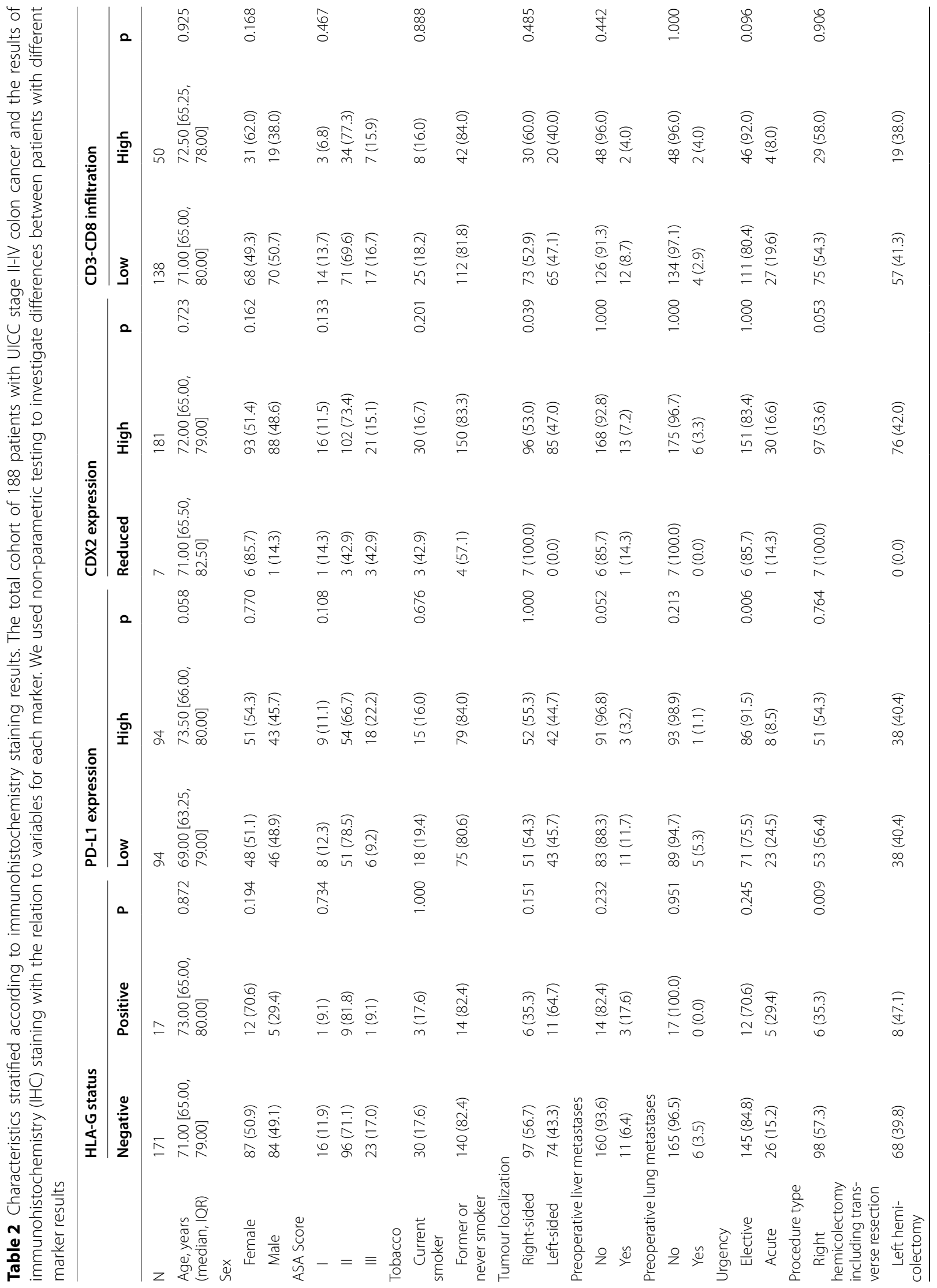




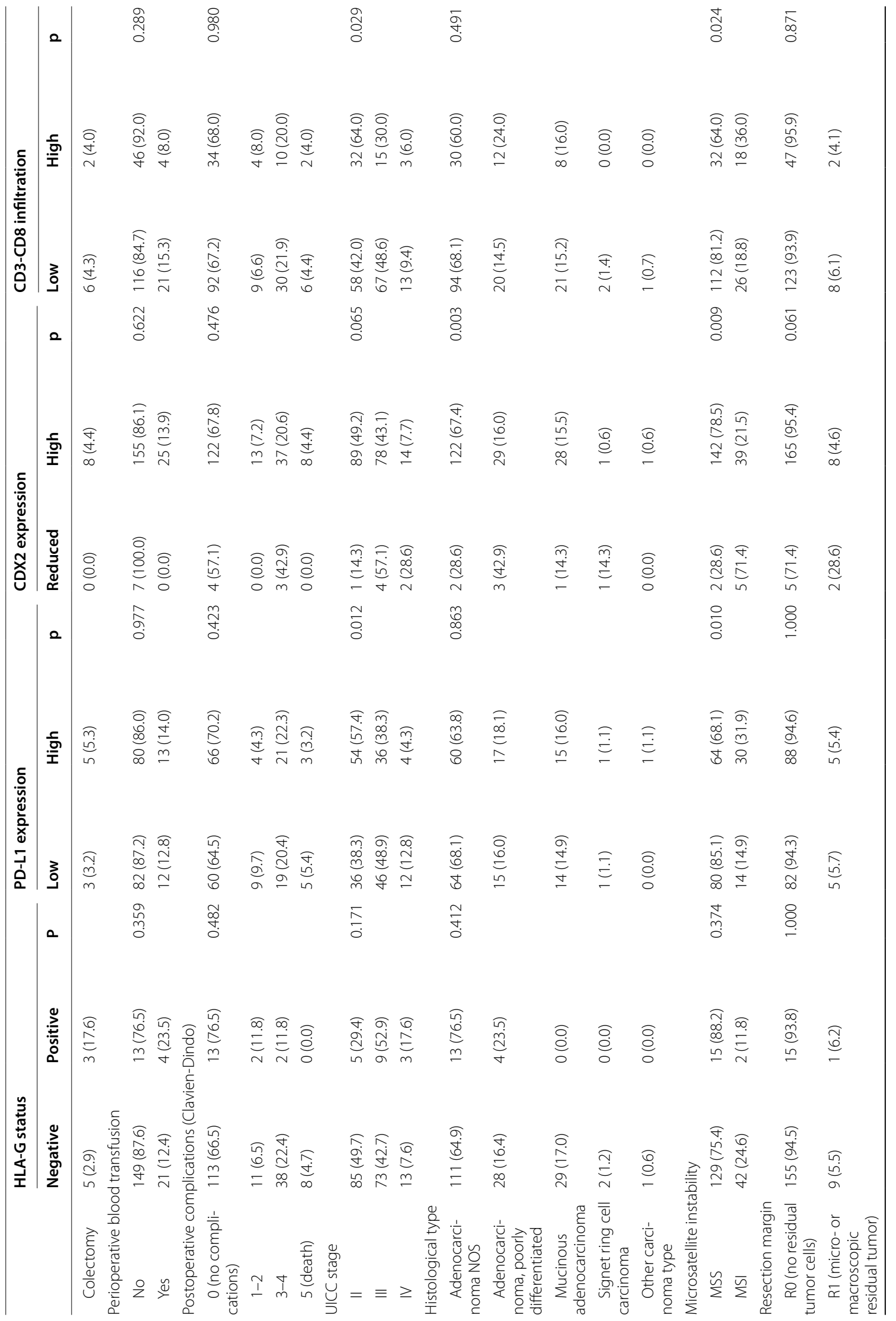




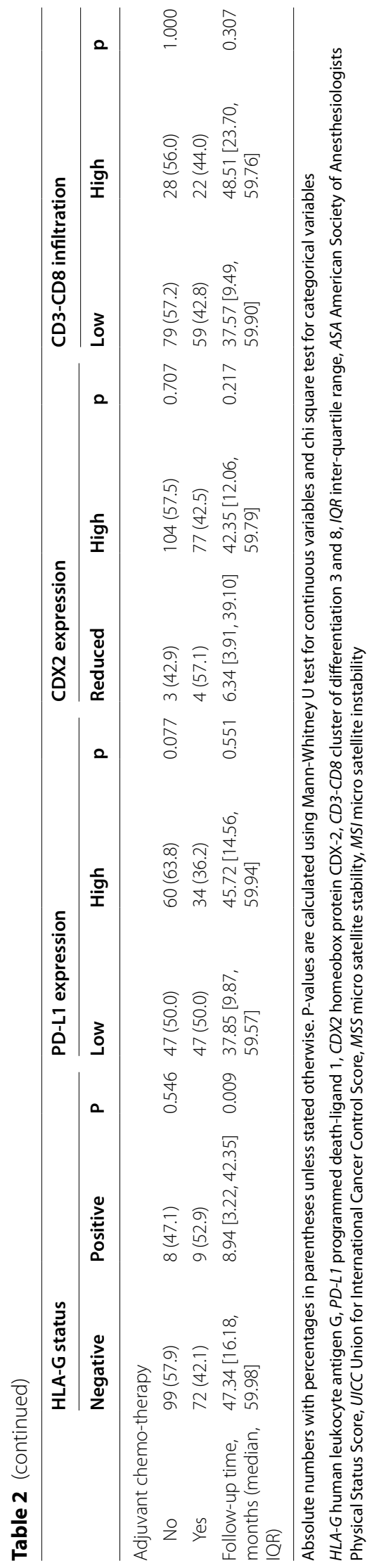




\section{PD-L1 expression status}

The median percentage of positive PD-L1 cells was 1.15\% (IQR 0.68-2.33\%) in the total cohort (Supplementary Table 1). Thirty (31.9\%) patients with high PD-L1 expression were MSI, while 14 (14.9\%) patients with low PD-L1 expression were MSI. A significant difference between PD-L1 expression and microsatellite status was found $(p=0.010$, Table 2$)$.

In the group of patients with low PD-L1 expression, $27(28.7 \%)$ patients experienced recurrence and 44 (46.8\%) patients died. In comparison, in the group with high PD-L1 expression 14 (14.9\%) events of recurrence occurred, and 30 (31.9\%) events of death were registered. In the non-parametric and unadjusted analyses there was no significant differences between groups for recurrence ( $p=0.067$, Fig. 3$)$ and OS ( $p=0.072$, Fig. 5), while a significant difference was found between groups for DFS ( $p=0.019$, Fig. 4). Multivariate regression analyses adjusted for confounders yielded lower but nonsignificant recurrence rates in the group of patients categorized as high expression of PD-L1, HR $=0.74$ (95\%CI [0.37-1.47], Fig. 6). The regression analyses for DFS and OS were not significant $(\mathrm{HR}=0.66,95 \% \mathrm{CI}$ [0.42-1.05] and $\mathrm{HR}=0.72,95 \% \mathrm{CI}$ [0.44-1.19], respectively, Figs. 7 and 8).

\section{CDX2 expression status}

Only seven (3.7\%) patients had reduced CDX2 expression of which five were MSI and two MSS. CDX2 expression was found to be significantly different based on microsatellite status $(p=0.009)$. Three patients with reduced CDX2 expression had poorly differentiated tumours compared with 29 patients with high CDX2 expression (42.9 and $16.0 \%$, respectively, $p=0.003$, Table 2 ).

The unadjusted non-parametric analyses between groups yielded a non-significant $p$-value for recurrence $(p=0.058$, Fig. 3$)$, and for DFS and OS $(p=0.081$ and $p=0.185$, respectively, Figs. 4 and 5). High CDX2 expression was associated with a significantly lower recurrence rate, $\mathrm{HR}=0.23$ (95\%CI [0.06-0.85], Fig. 6), in the confounder adjusted multivariable regression analysis. The regression analyses for DFS and OS were not significant $(\mathrm{HR}=0.57,95 \% \mathrm{CI}[0.21-1.58]$ and $\mathrm{HR}=0.87,95 \% \mathrm{CI}$ [0.33-2.30], respectively, Figs. 7 and 8).

\section{CD3 and CD8 expression status}

The median percentage of CD3-positive cells in the tumour centre was 13.34\% (IQR 8.46-21.05) and 18.16\% (IQR 11.31-24.05) in the invasive margin. The median percentage of CD8-positive cells in the tumour centre was 6.11\% (IQR 3.08-11.13) and 9.32\% (IQR 5.59$14.10)$ in the invasive margin. The merged CD3-CD8 score yielded 138 (73.4\%) low infiltrated tumours and 50 (26.6\%) high infiltrated tumours (Supplementary Table 1). Eighteen (36\%) patients with high infiltrated tumours were MSI, while $26(18.8 \%)$ patients with low infiltrated tumours were MSI, and the difference was significant ( $p=0.024$, Table 2$)$.

The unadjusted non-parametric analyses found no significant difference between groups for recurrence $(p=0.167$, Fig. 3), while a significant difference was found for DFS and OS $(p=0.027$ and $p=0.031$, respectively, Figs. 4 and 5). Confounder adjusted multivariable regression analysis did not show a significant lower recurrence rate for patients with a high CD3-CD8 score $(\mathrm{HR}=0.72$, 95\%CI [0.33-1.60], Fig. 6). However, this group of patients did have a significantly longer DFS, $\mathrm{HR}=0.55(95 \% \mathrm{CI}$ [0.31-0.98], Fig. 7) and a significantly longer OS, $\mathrm{HR}=0.53$ (95\%CI [0.29-0.99], Fig. 8).

\section{Combined marker score}

A combined IHC score of all markers resulted in 37 (19.7\%) patients with a low score, 139 (73.9\%) patients with an intermediate score, and $12(6.4 \%)$ patients with a high score (Supplementary Table 1).

In the unadjusted non-parametric analyses there were significant differences between the three groups for recurrence $(p<0.001$, Fig. 3$)$, DFS ( $p=0.001$, Fig. 4$)$ and OS ( $p=0.003$, Fig. 5). Confounder adjusted multivariable regression analyses yielded a significantly higher recurrence rate for patients with an intermediate and a high combined marker score $(\mathrm{HR}=7.56,95 \% \mathrm{CI}$ [1.06-54.16] and $\mathrm{HR}=20.53,95 \% \mathrm{CI}$ [2.68-157.32], respectively, Fig. 6). An intermediate and a high combined marker score were associated with a significantly shorter DFS $(\mathrm{HR}=2.85,95 \% \mathrm{CI}[1.28-6.31]$ and $\mathrm{HR}=5.27,95 \% \mathrm{CI}$ [1.95-14.29], respectively, Fig. 7) compared with a low score. An intermediate and a high combined marker score were associated with a significantly shorter OS

(See figure on next page.)

Fig. 3 Cumulative incidence plots of recurrence. $P$-values are estimated using Gray's test. Time-to-recurrence after colon cancer resection stratified by expression of HLA-G, PD-L1, CDX2 and the CD3-CD8 score and the combined marker score. The combined marker score was computed based on the expression of the markers. Score 0 represents a low combined marker score indicating a favourable prognosis, 1 represents an intermediate combined marker score, and 2 represents a high combined marker score indicating an unfavourable prognosis 

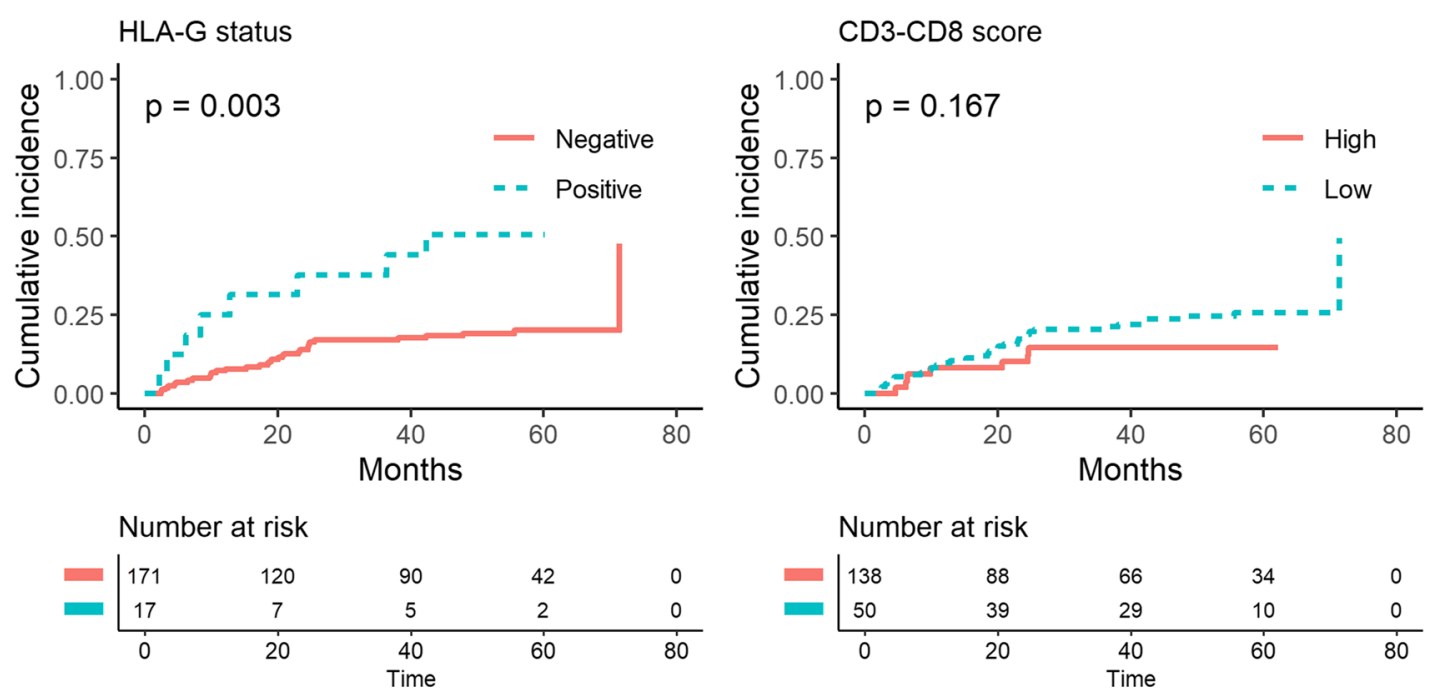

PD-L1 expression

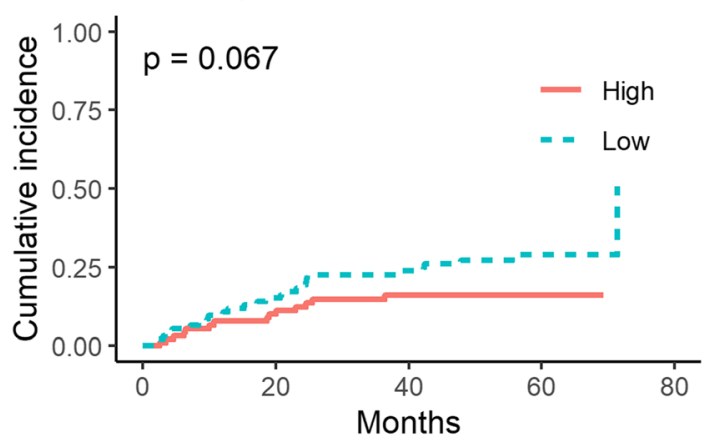

Combined marker score

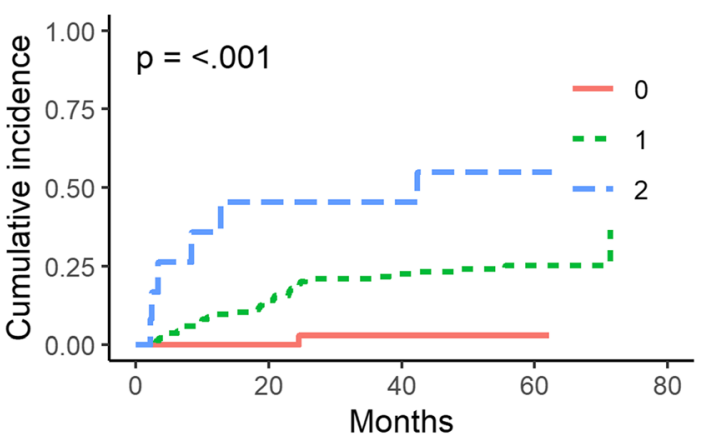

Number at risk

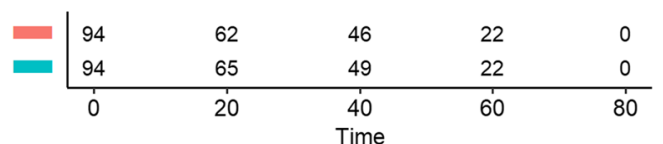

Number at risk

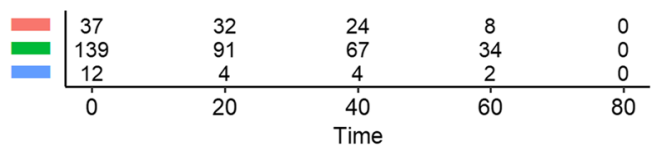

CDX2 expression

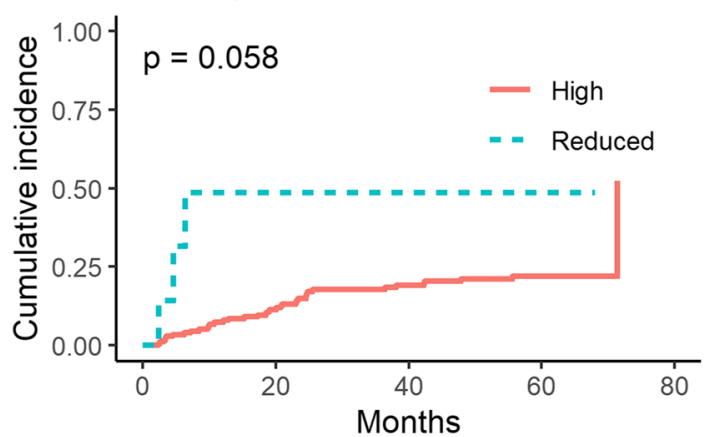

Number at risk

$=\mid$\begin{tabular}{ccccc}
7 & 2 & 2 & 1 & 0 \\
181 & 125 & 93 & 43 & 0 \\
\hline 0 & 20 & 40 & 60 & 80
\end{tabular}

Fig. 3 (See legend on previous page.) 
$(\mathrm{HR}=2.72,95 \% \mathrm{CI}[1.14-6.46]$ and $\mathrm{HR}=4.00,95 \% \mathrm{CI}$ [1.38-11.53], respectively, Fig. 8) compared with a low score in a confounder adjusted multivariate analysis.

\section{Discussion}

In this study, we explored the expression of prognostic markers in patients with pT3 and pT4 colon cancers including HLA-G and PD-L1, two markers of immune evasion, as well as the expression of CDX2, a marker of differentiation, and CD3 and CD8, markers of TILs. In adjusted multivariable Cox regression models, positive HLA-G expression was associated with a shortened time to recurrence while a preserved CDX2 expression was associated with a prolonged time to recurrence. When we combined all IHC markers into a summarized score of an unfavourable expression pattern, we found an intermediate and a high combined marker score to be associated with a shortened time to recurrence.

Our results of HLA-G expression as a prognostic marker are in accordance with previously published studies on patients with colorectal cancer [14-17]. HLA-G expression has also been shown to be associated with a shortened time to recurrence, DFS and OS in several other malignancies such as gastric cancer, breast cancer, lung cancer and malignant melanoma [30-33]. During pregnancy, HLA-G modulates the maternal immune response to accept the semi-allogenic foetus [34, 35]. These results are all in accordance with a pathophysiological expression of HLA-G and its modulatory effects on cells of the immune system [10-13]. We defined HLA-G-positive tumours as 10 or more positive cells in one full slide, which may be a very low cut-off. The literature is sparse and divergent on survival analyses and cut-off values for HLA-G expression. Dichotomising HLA-G expression based on positive expression $(>0 \%$ positive cells) or a 5\%-cut-off has previously been used in prognostic biomarker studies on patients with colorectal cancer [15-17, 36, 37]. We had a lower occurrence of HLA-G-positive tumours than the published studies with a $>0 \%$ cut-off with $9.0 \%$ in our cohort compared with 70.6 and $65 \%$ in two Chinese populations and $20.3 \%$ in a Dutch population, thereby in more accordance with our study $[16,17,36]$. The Dutch study utilized the same antibody (4H84) as we did while using tissue microarrays (TMAs) instead of evaluating full slides. The $4 \mathrm{H} 84$
mAb detects denatured HLA-G molecules. The authors included patients with colon cancer of all T-stages, although their population had primarily T3 tumours, while we in the present study only included patients with T3 and T4 colon cancer tumours [36]. The two Chinese studies both evaluated full slides; however, different anti-HLA-G antibodies were used; the MEM-G/2 mAB, which binds free heavy chain of all HLA-G isoforms, and an anti-HLA-G mAb (HGY) not available commercially that should detect both membrane and soluble HLA-G isoforms. The studies included patients with colon and rectal cancer with all T-stages. The study with the highest proportion of patients with HLA-G positive tumours did not stratify patients in colon and rectal cancer cohorts [16]. However, the other Chinese study found a lower proportion of HLA-positivity in patients with rectal cancer [17]. Direct comparison does not seem possible due to the different methods applied in these studies compared to ours e.g. TMA versus full slides and different antibodies applied. Furthermore, both inter- and intratumour heterogeneity have been reported for HLA-G in colorectal tumours $[38,39]$. Thus, the expression of HLA-G varies depending on the location within the tumour. The inconsistent HLA-G findings across studies could be attributed to several factors. A number of different anti-HLA-G mAbs are used in the published studies, one (HGY) is not commercially available and staining specifities seem not to have been widely assessed. The mAbs may bind to different epitopes, which may influence the detection rate of HLA-G isoform expression in different tumours. It can be speculated that there might also be ethnic differences; the percentages of tumours expressing HLA-G are closest within the two studies including Caucasian patient groups and within the two studies including Asian patient groups, respectively. Furthermore, novel alternatively spliced HLA-G isoforms have been characterized in clear cell renal cell carcinoma specimens, which may theoretically also occur in colon cancers and influence the staining patterns [40]. Finally, even with the same population and IHC methods, formalin fixation time has been shown to affect the IHC reactions [41]. Interestingly, HLA-G may be a potential new therapeutic target for cancer immunotherapy [42]. One study utilizing chimeric antigen receptor T-cells (CAR-T cells) directed against HLA-G was recently published,

(See figure on next page.)

Fig. 4 Kaplan-Meier plots of Disease-Free Survival. Disease-Free Survival (DFS) after colon cancer resection stratified by expression of HLA-G, PD-L1, CDX2, and CD3-CD8 score and combined marker score. The combined marker score was computed based on the expression of the markers. Score 0 represents a low combined marker score indicating a favourable prognosis, 1 represents an intermediate combined marker score, and 2 represents a high combined marker score indicating an unfavourable prognosis. P-values were estimated using log-rank test 

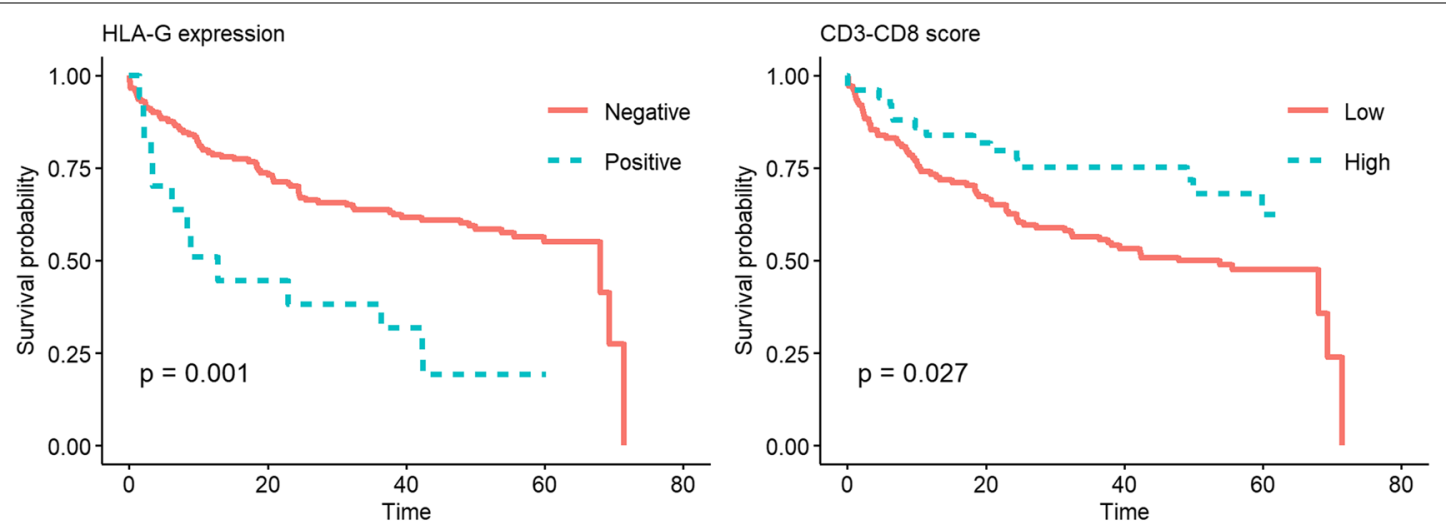

Number at risk

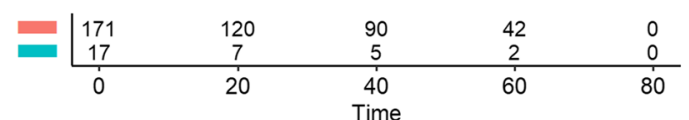

Number at risk
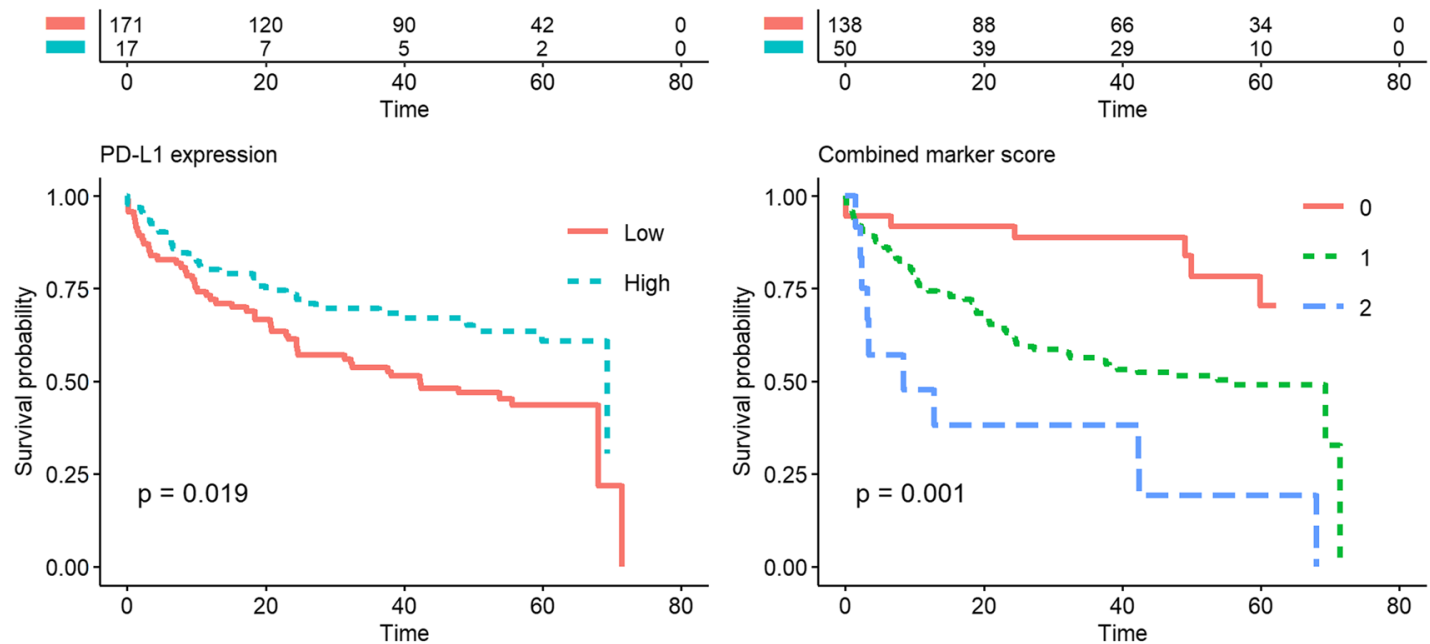

Combined marker score

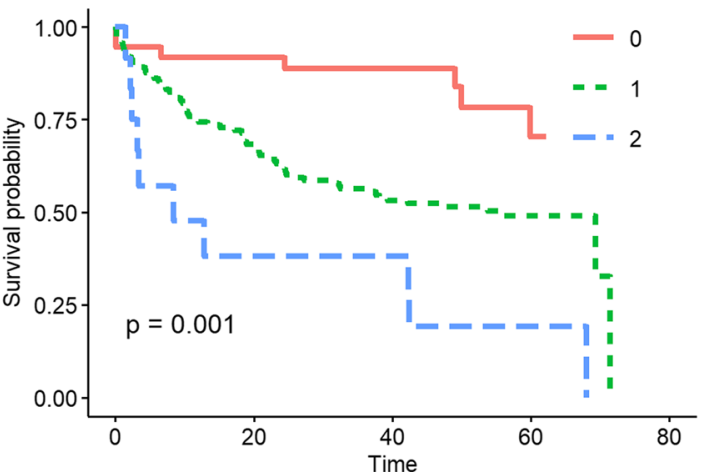

Number at risk

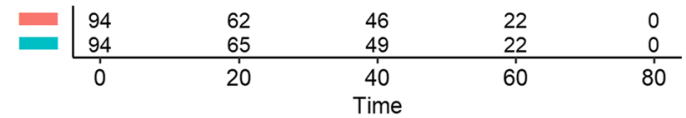

Number at risk

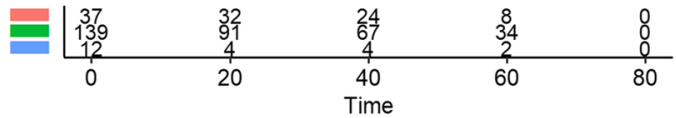

CDX2 expression

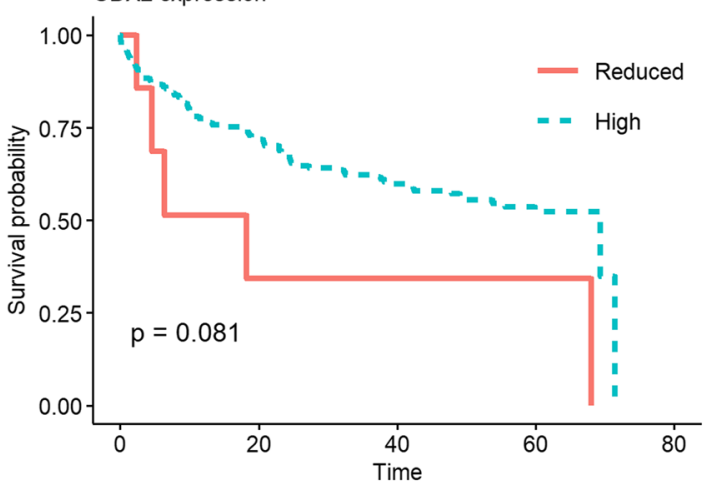

Number at risk

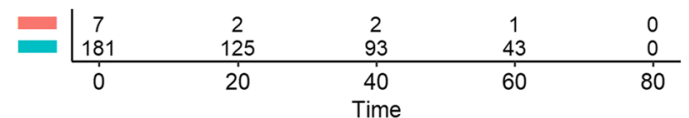

Fig. 4 (See legend on previous page.) 
while a number of patents have been filed for experimental antibodies directed against HLA-G and its receptors $[43,44]$.

A consensus guideline for assessment of PD-L1 has not been established for colon cancer. We used a combined positive score for cancer cells and immune cells expressing PD-L1 as a surrogate marker of immune activation. We found patients with high PD-L1 expression to have a longer DFS in unadjusted non-parametric analyses. Four studies based on TMAs have investigated the combined expression of PD-L1 in tumour and immune cells in patients with colorectal cancer [24, 45-47]. All four studies did find an association of a high combined PD-L1 expression and longer survival, however, they used different antibodies and performed manual assessment of the PD-L1 stainings. A recent meta-analysis of PD-L1 expression and prognosis in patients with colorectal cancer did not recommend PD-L1 as a prognostic marker even though the conclusion was that immune cell expression of PD-L1 was associated with a better survival [48]. As PD-L1 expression may be a marker of good prognosis when expressed by immune cells, and may be a marker of bad prognosis when expressed by tumour cells, it might be more informative not to use a combined positive score as we did, but differentiate between the cell types [23, 24]. However, our analytic platform did not allow for this distinguishment.

CDX2 is a gastrointestinal-specific transcription factor [49]. We identified only $3.7 \%$ of our cohort with a reduced CDX2 expression. Patients with reduced CDX2 expression had significantly shortened time to recurrence. Previously, loss of CDX2 has been described as strongly associated with poor prognosis in patients with colorectal cancer $[25,50,51]$. Our results support that loss of CDX2 is a marker of poorly differentiated tumours. Furthermore, we found a reduced CDX2 expression to be associated with MSI status. Interestingly, a study reported that loss of CDX2 expression could predict survival only in patients with MSS [51]. Loss of CDX2 expression has also been suggested to identify a high-risk subgroup of patients with stage II [25].

In our study, patients with a high CD3-CD8-score had a significantly prolonged DFS as well as a prolonged OS. Thus, our results are in line with those shown for the Immunoscore $\odot$ in several publications and cohorts of patients with colorectal cancer $[7,8,52]$. We did not follow the Immunoscore $\odot$ protocol, as we used percentages of positive cells instead of densities, different antibodies, laboratory equipment and software for digital analysis. We did, however, adopt a similar approach when calculating a score for TILs, based on digital counts of CD3- and CD8-positive cells in two tumour compartments (the tumour centre and the invasive margin). Patients with early stage disease, UICC stage I, have been found to have a higher infiltration of TILs than patients with UICC stage II-IV [53]. We did not include patients with UICC stage I, but we did, however, find patients with a high CD3-CD8 score to have a higher occurrence of UICC stage II disease than patients with a low CD3-CD8 score. We also found patients with a high CD3-CD8 score to have a higher occurrence of MSI than patients with a low CD3-CD8 score. Accordingly, tumours with MSI are associated with a high immune system activation due to the high expression of tumour-associated antigens [7].

When we combined all our markers into a combined marker score, we identified the strongest signal in the regression analyses. Both an intermediate and a high combined marker score were significantly associated with an increased risk of recurrence and mortality. Our data confirmed that a combination of prognostic markers could provide a stronger estimate of prognosis. A previous study combining the results of HLA class I- and FoxP3-expression based on a computed immune phenotype, could identify a distinct survival pattern between three different phenotypes [36]. The width of the $95 \% \mathrm{CI}$ in our study, reveals that our HR should be interpreted with great care. When calculating the score, all markers contributed with the same weight to the total score. However, this may not be the optimal approach as each marker may contribute differently to the risk of recurrence or death.

A strength of this study is inclusion of consecutive patients during a two-year inclusion span. We chose to focus on patients with pT3 and pT4 tumours in the colon based on the higher risk of recurrence [54]. In all tumour samples, the invasive margin was represented and assessment was performed on full slides. We investigated the expression of more than one immune checkpoint in patients with colon cancer, and each patient was analysed for TILs. Apart from the previously mentioned limitations only a low number of patients with reduced CDX2 expression $(n=7)$ and positive HLA-G expression $(n=17)$ were

(See figure on next page.)

Fig. 5 Kaplan-Meier plots of Overall Survival. Overall Survival (OS) after colon cancer resection stratified by expression of HLA-G, PD-L1, CDX2, and CD3-CD8 score and combined marker score. The combined marker score was computed based on the expression of the markers. Score 0 represents a low combined marker score indicating a favourable prognosis, 1 represents an intermediate combined marker score, and 2 represents a high combined marker score indicating an unfavourable prognosis. P-values were estimated using log-rank test 

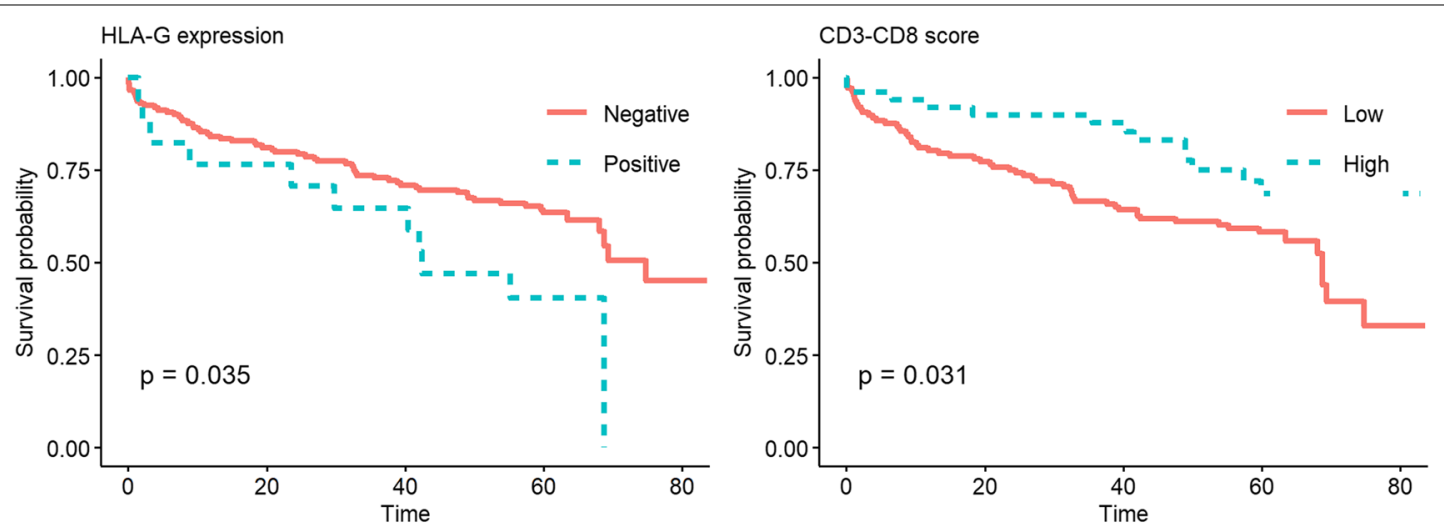

Number at risk

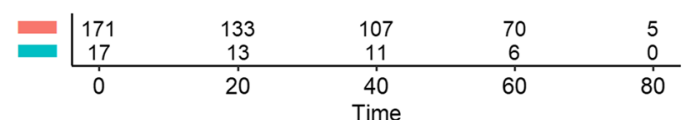

Number at risk
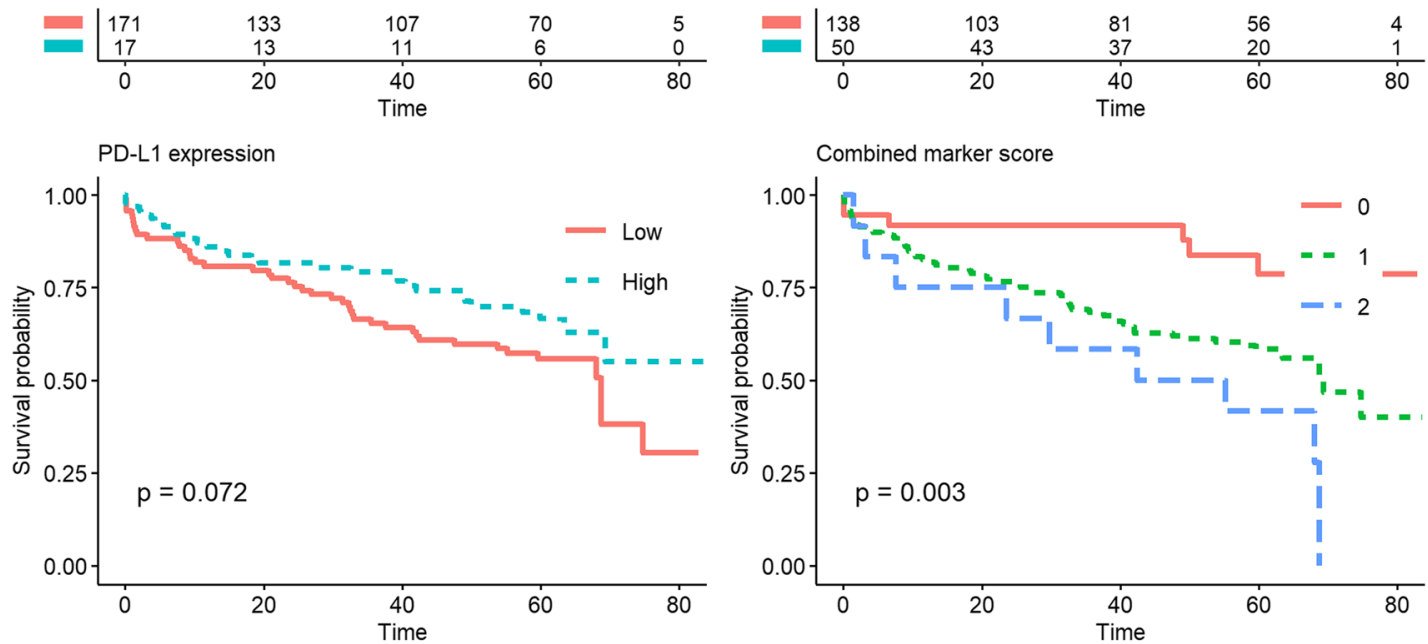

Combined marker score

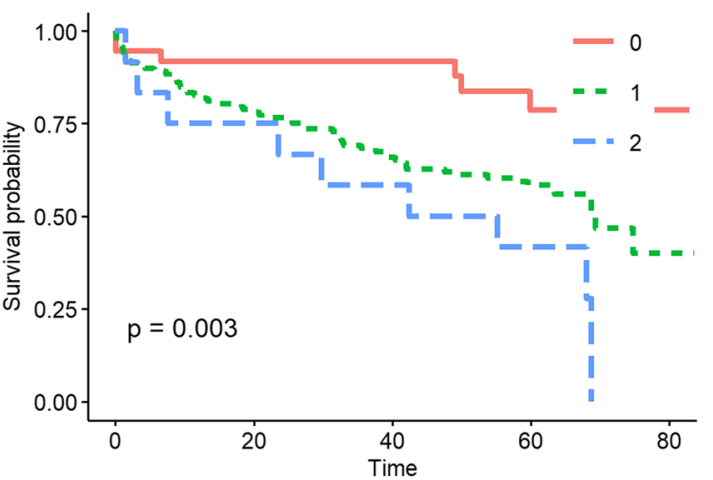

Number at risk

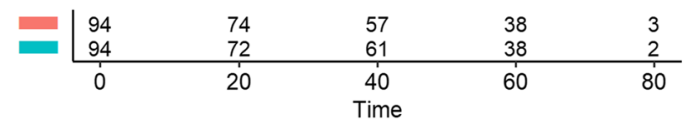

Number at risk

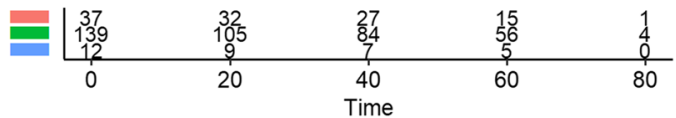

CDX2 expression

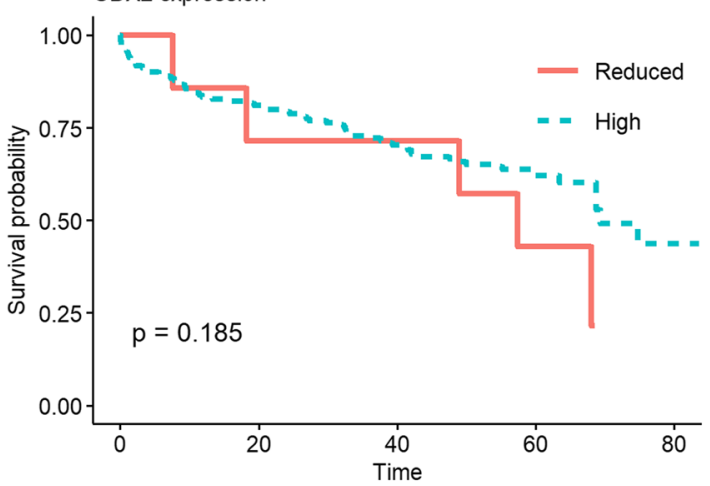

Number at risk

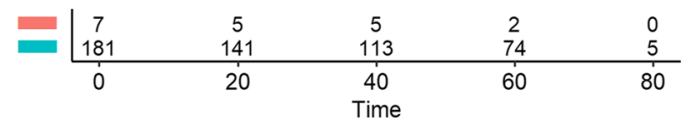

Fig. 5 (See legend on previous page.) 


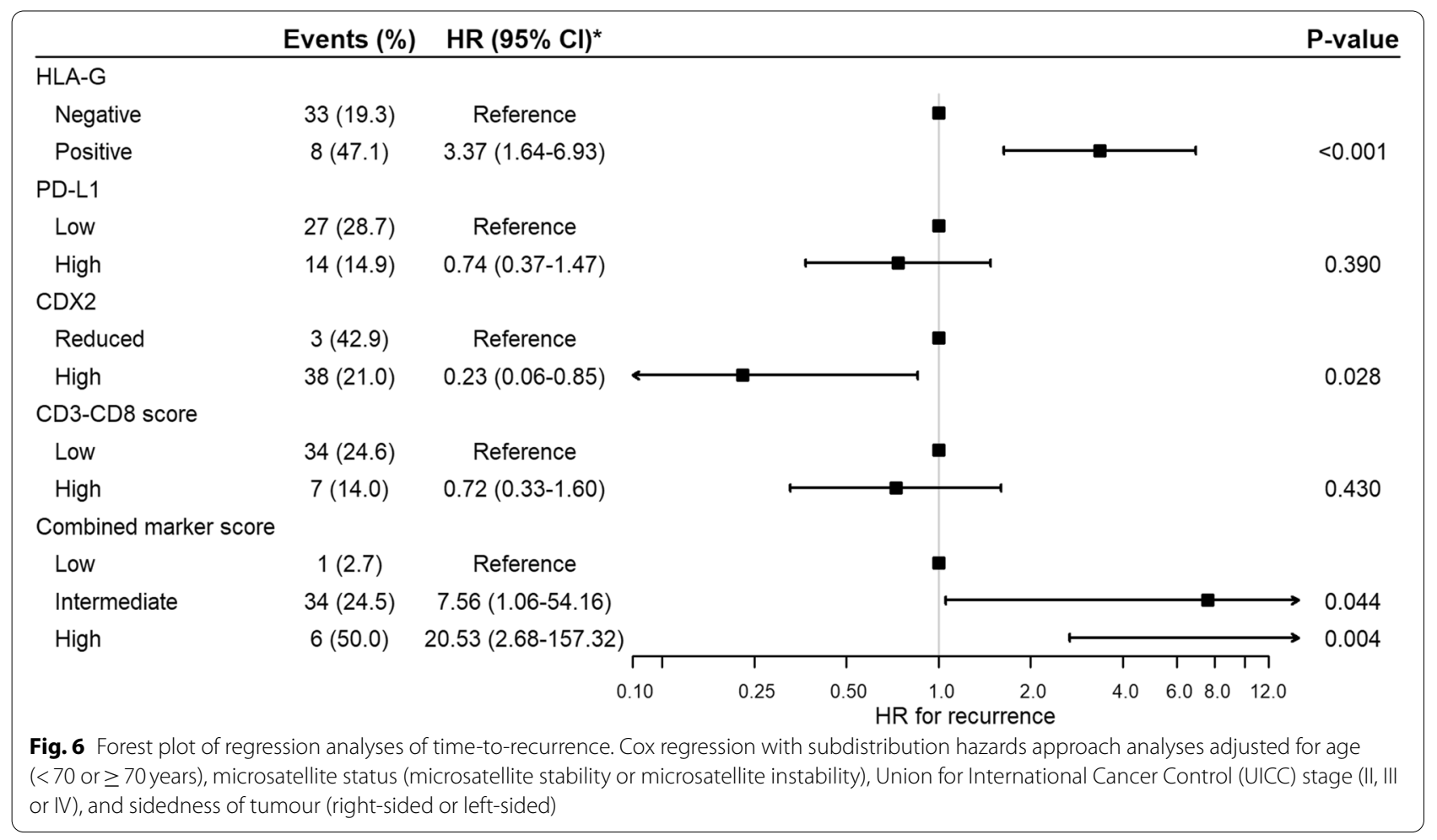

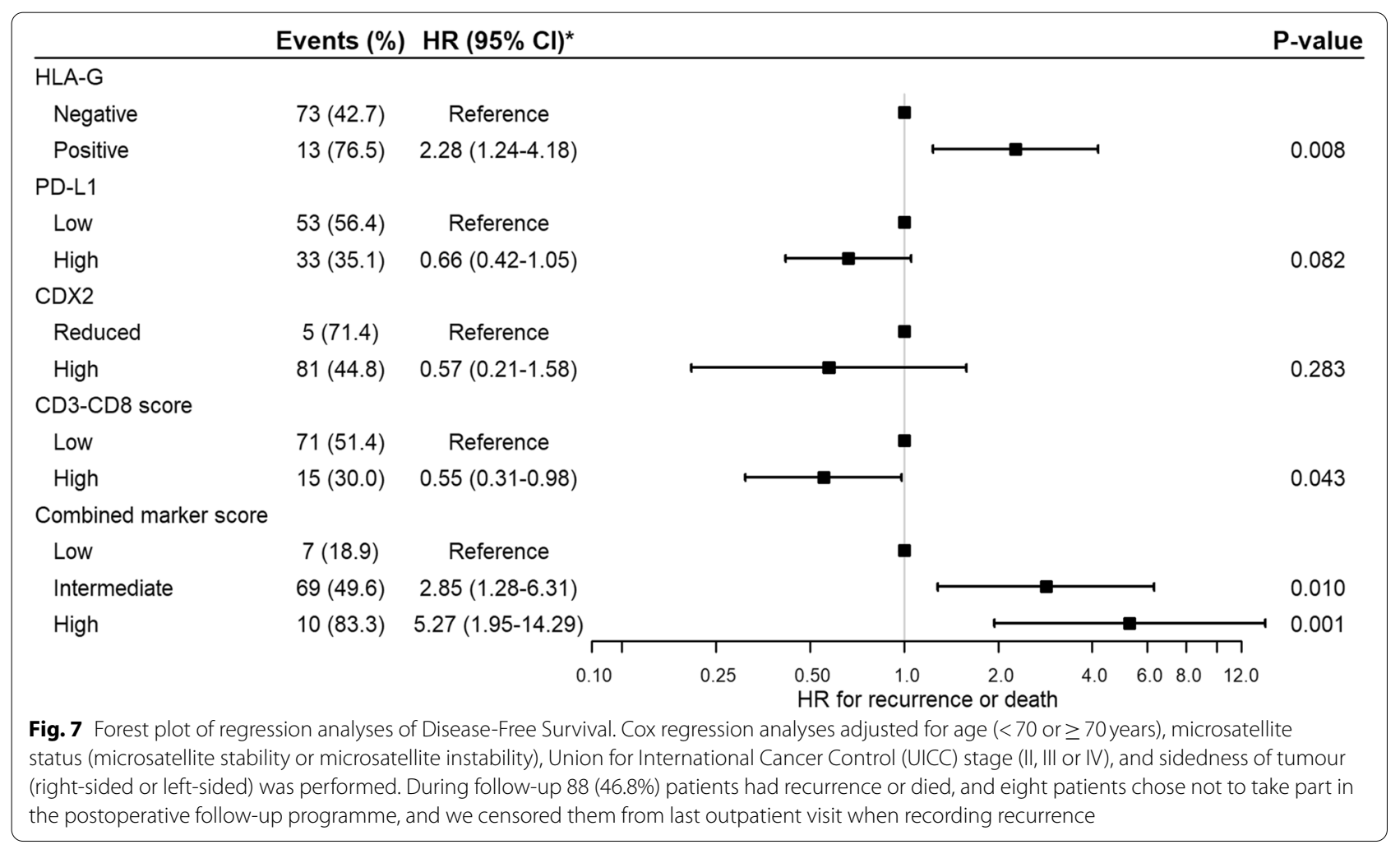




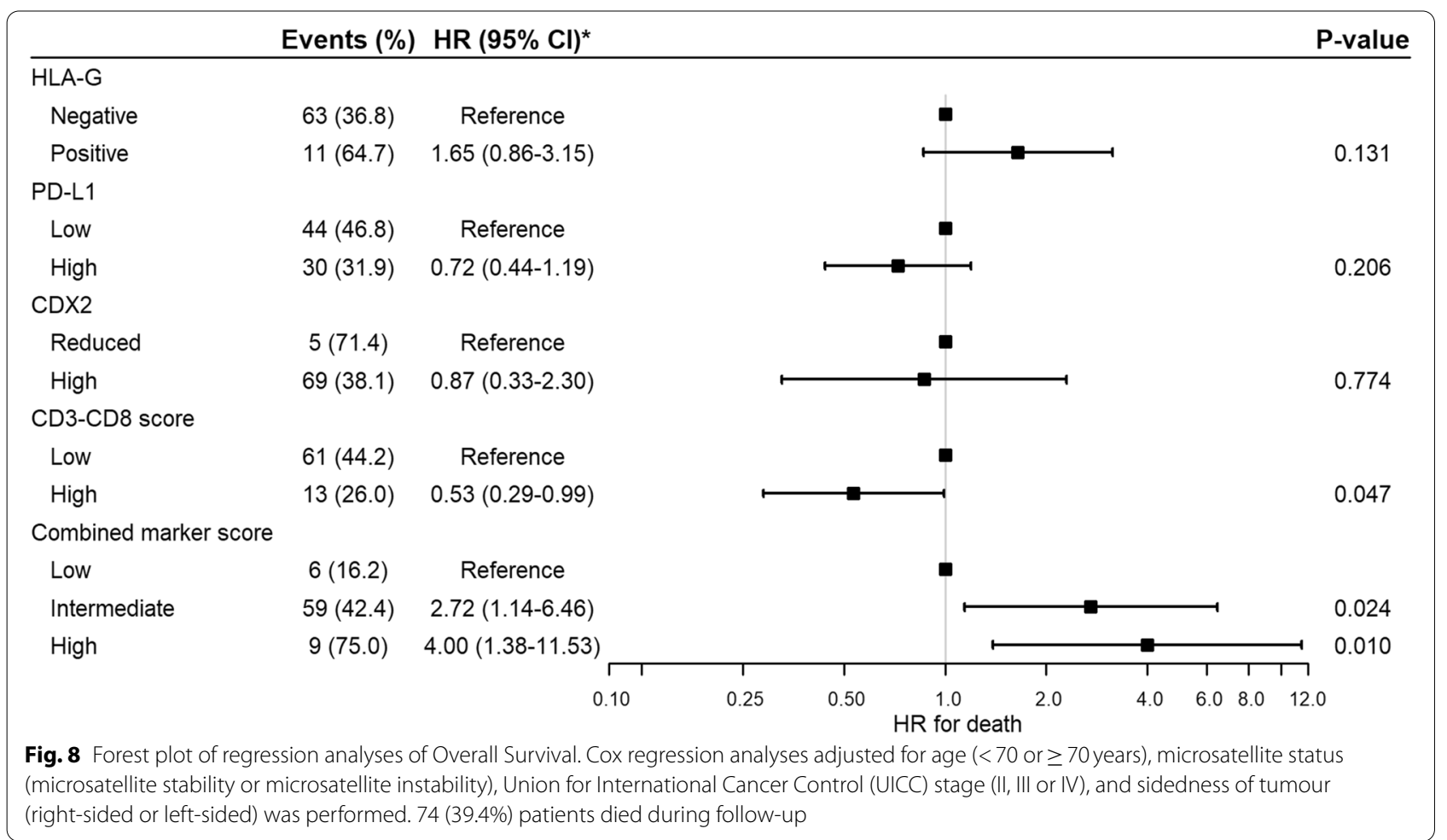

identified. This resulted in limited statistical power in our analyses. We saw the strongest signal in the time-to-recurrence analyses. Time-to-recurrence comes with a threat of competing risk bias in case patients die before they can develop recurrence [55]. We attempted to reduce this risk by applying the Fine-Gray method.

\section{Conclusions}

In conclusion, we investigated HLA-G, PD-L1, CDX2, and CD3 and CD8 as prognostic markers in patients with pT3 and pT4 colon cancers. We found positive HLA-G expression, and a high combined marker score to be independently associated with a shortened time to recurrence. Preserved expression of CDX2 was independently associated with a longer time to recurrence.

\footnotetext{
Abbreviations

ASA: American Society of Anaesthesiologists; CAR-T cells: Chimeric antigen receptor T-cells; CD3: Cluster of differentiation 3; CD8: Cluster of differentiation 8; Cl: Confidence intervals; DFS: Disease-free survival; FFPE: Formalin-fixed paraffin-embedded; H\&E: Haematoxylin and eosin; HLA: Human leukocyte antigen; HLA-G: Human leukocyte antigen G; HR: Hazard ratios; IHC: Immunohistochemical; IQR: Inter-quartile ranges; MSI: Microsatellite instable; MSS: Microsatellite stabile; NK cells: Natural killer cells; NOS: Not otherwise specified; OS: Overall survival; PD-1: Programmed death 1; PD-L1: Programmed deathligand 1;TILs: Tumour-infiltrating lymphocytes; UICC: Union for International Cancer Control.
}

\section{Supplementary Information}

The online version contains supplementary material available at https://doi. org/10.1186/s12885-022-09169-0.

Additional file 1 : Supplementary Table 1. PD-L1, CD3 and CD8 analyses and calculation of scores.

\section{Acknowledgements}

We would like to thank the staff at the Department of Pathology, Zealand University Hospital and Næstved Hospital, for their dedication in performing the assays and analyses. We thank consultant surgeon Henrik Ovesen for his support in the collection of patient data. Also, the team at Center for Surgical Science, and especially Anne Dalsgaard Hansen and Tove Kirkegaard Clausen, for their dedication in helping with administrative tasks.

\section{Authors' contributions}

$A B, S E, M B, T H$ and IG contributed to the conception and the design of the study. AB collected patient data from patient files, assessed CDX2 stained slides, prepared scanned CD3, CD8, and PD-L1 stained slides for digital counting. AB combined the datasets and calculated the scores as well as interpreting the results and writing the first draft of the manuscript. LC, AA, KM collected substantial patient data from patient files. JE performed the digital counting of CD3, CD8, and PD-L1 stained slides and contributed to the interpretation of IHC data. SE performed the histological evaluation of all HE slides for selection of FFPE tissue samples as well as assessing the CDX2 and HLA-G stained slides. MB performed all IHC staining as well as optimizing the staining-protocols for each marker. AF prepared scanned CD3, CD8, and PD-L1 stained slides for digital counting and assessed the HLA-G stained slides manually as well as contributing substantially in the interpretation of data and in the writing of the manuscript. TH contributed to the HLA-G staining protocol and the analyses and interpretation of HLA-G data as well as being a major contributor in writing the manuscript. 
IG contributed substantially during the acquisition of data, the analyses, interpretation of patient and IHC data, and was a major contributor in writing the manuscript. RH performed all survival statistics and was a substantial contributor in the interpretation and drafting of the manuscript. All authors read and approved the final manuscript.

\section{Funding}

This study was supported by the Novo Nordisk Foundation [2015: 137,660 DKKR], the Foundation of 17-12-1981 [2015, 50,000 DKKR], and the Region Zealand Health Research Foundation [15-000342, 270,000 DKKR]. The foundations had no role in the design, collection, analyses or interpretation of data as well as no role in writing the manuscript. The Department of Clinical Biochemistry and the Department of Surgery provided funding from departmental sources.

\section{Availability of data and materials}

The datasets used and analysed during the current study are available from the corresponding author on reasonable request.

\section{Declarations}

\section{Ethics approval and consent to participate}

The study was approved by the Regional Ethics Committee of Region Zealand (SJ-589) and the Data agency (REG-124-2016), and was exempted from the rule of informed consent. Patients registered in the Danish Registry for Use of Tissue (refusing to have their tissue used in research) was excluded from the study. All study methods were carried out in accordance with the Declaration of Helsinki.

\section{Consent for publication}

Not applicable.

\section{Competing interests}

None of the authors has any financial or non-financial relationship with organizations that could inappropriately influence this study.

IG has received unrestricted research grants from Intuitive Surgical, Perfusion Tech, Pharmacosmos, and Reponex Pharmaceuticals A/S, and consultancy fees from Ethicon and Medtronic.

\begin{abstract}
Author details
${ }^{1}$ Center For Surgical Science (CSS), Department of Surgery, Zealand University Hospital, Lykkebækvej 1, 4600 Køge, Denmark. ${ }^{2}$ Cardiology department, Hospital Sønderjylland, Kresten Philipsens Vej 15, 6200 Aabenraa, Denmark. ${ }^{3}$ Department of Pathology, Zealand University Hospital, Sygehusvej 10, 4000 Roskilde, Denmark. ${ }^{4}$ Department of Clinical Medicine, University of Copenhagen, Blegdamsvej 3b, 2200 Copenhagen, Denmark. ${ }^{5}$ Centre for Immune Regulation and Reproductive Immunology (CIRRI), Department of Clinical Biochemistry, Zealand University Hospital, Sygehusvej 10, 4000 Roskilde, Denmark.
\end{abstract}

Received: 9 September 2021 Accepted: 23 December 2021 Published online: 14 January 2022

\section{References}

1. Hanahan D, Weinberg RA. Hallmarks of cancer: the next generation. Cell. 2011;144(5):646-74.

2. Fridman WH, Zitvogel L, Sautès-Fridman C, Kroemer G. The immune contexture in cancer prognosis and treatment. Nat Rev Clin Oncol. 2017;14(12):717-34.

3. Chen DS, Mellman I. Elements of cancer immunity and the cancer-immune set point. Nature. 2017;541(7637):321-30.

4. Dunn GP, Old $\amalg$, Schreiber RD. The three Es of cancer immunoediting. Annu Rev Immunol. 2004;22:329-60.

5. Lakatos E, Williams MJ, Schenck RO, Cross WCH, Househam J, Zapata L, et al. Evolutionary dynamics of neoantigens in growing tumors. Nat Genet. 2020;52(10):1057-66.
6. Le DT, Uram JN, Wang H, Bartlett BR, Kemberling H, Eyring AD, et al. PD-1 blockade in tumors with mismatch-repair deficiency. N Engl J Med. 2015;372(26):2509-20.

7. Mlecnik B, Bindea G, Angell HK, Maby P, Angelova M, Tougeron D, et al. Integrative analyses of colorectal cancer show immunoscore is a stronger predictor of patient survival than microsatellite instability. Immunity. 2016;44(3):698-711.

8. Mlecnik B, Tosolini M, Kirilovsky A, Berger A, Bindea G, Meatchi T, et al. Histopathologic-based prognostic factors of colorectal cancers are associated with the state of the local immune reaction. J Clin Oncol. 2011;29(6):610-8.

9. Garrido F, Cabrera T, Concha A, Glew S, Ruiz-Cabello F, Stern PL. Natural history of HLA expression during tumour development. Immunol Today. 1993;14(10):491-9.

10. Morandi F, Rizzo R, Fainardi E, Rouas-Freiss N, Pistoia V. Recent advances in our understanding of HLA-G biology: lessons from a wide spectrum of human diseases. J Immunol Res. 2016;2016:4326495.

11. Le Gal FA, Riteau B, Sedlik C, Khalil-Daher I, Menier C, Dausset J, et al. HLAG-mediated inhibition of antigen-specific cytotoxic T lymphocytes. Int Immunol. 1999;11(8):1351-6.

12. Naji A, Menier C, Morandi F, Agaugué S, Maki G, Ferretti E, et al. Binding of HLA-G to ITIM-bearing lg-like transcript 2 receptor suppresses B cell responses. J Immunol. 2014;192(4):1536-46.

13. Pazmany L, Mandelboim O, Valés-Gómez M, Davis DM, Reyburn HT, Strominger JL. Protection from natural killer cell-mediated lysis by HLA-G expression on target cells. Science. 1996;274(5288):792-5.

14. Reimers MS, Engels CC, Putter H, Morreau H, Liefers GJ, van de Velde CJ, et al. Prognostic value of HLA class I, HLA-E, HLA-G and Tregs in rectal cancer: a retrospective cohort study. BMC Cancer. 2014;14:486.

15. Zhang RL, Zhang X, Dong SS, Hu B, Han QY, Zhang JG, et al. Predictive value of different proportion of lesion HLA-G expression in colorectal cancer. Oncotarget. 2017;8(64):107441-51.

16. Guo ZY, Lv YG, Wang L, Shi SJ, Yang F, Zheng GX, et al. Predictive value of HLA-G and HLA-E in the prognosis of colorectal cancer patients. Cell Immunol. 2015;293(1):10-6.

17. Ye SR, Yang H, Li K, Dong DD, Lin XM, Yie SM. Human leukocyte antigen G expression: as a significant prognostic indicator for patients with colorectal cancer. Modern Pathol. 2007;20(3):375-83.

18. Patsoukis N, Wang Q, Strauss L, Boussiotis VA. Revisiting the PD-1 pathway. Sci Adv. 2020;6(38):eabd2712.

19. Francisco LM, Sage PT, Sharpe AH. The PD-1 pathway in tolerance and autoimmunity. Immunol Rev. 2010;236:219-42.

20. Bocanegra A, Blanco E, Fernandez-Hinojal G, Arasanz H, Chocarro L, Zuazo M, et al. PD-L1 in systemic immunity: unraveling its contribution to PD-1/PD-L1 blockade immunotherapy. Int J Mol Sci. 2020;21 (16):5918.

21. Masugi Y, Nishihara R, Yang J, Mima K, da Silva A, Shi Y, et al. Tumour CD274 (PD-L1) expression and T cells in colorectal cancer. Gut. 2017;66(8):1463-73.

22. Watson MM, Lea D, Gudlaugsson E, Skaland I, Hagland HR, Søreide K. Prevalence of PD-L1 expression is associated with EMAST, density of peritumoral T-cells and recurrence-free survival in operable non-metastatic colorectal cancer. Cancer Immunol Immunother. 2020;69(8):1627-37.

23. Ho HL, Chou TY, Yang SH, Jiang JK, Chen WS, Chao Y, et al. PD-L1 is a double-edged sword in colorectal cancer: the prognostic value of PD-L1 depends on the cell type expressing PD-L1. J Cancer Res Clin Oncol. 2019;145(7):1785-94.

24. Bae SU, Jeong WK, Baek SK, Kim NK, Hwang I. Prognostic impact of programmed cell death ligand 1 expression on long-term oncologic outcomes in colorectal cancer. Oncol Lett. 2018;16(4):5214-22.

25. Dalerba P, Sahoo D, Paik S, Guo X, Yothers G, Song N, et al. CDX2 as a prognostic biomarker in stage II and stage III Colon cancer. N Engl J Med. 2016;374(3):211-22.

26. McShane LM, Altman DG, Sauerbrei W, Taube SE, Gion M, Clark GM. REporting recommendations for tumour MARKer prognostic studies (REMARK). Br J Cancer. 2005;93(4):387-91.

27. Røge R, Vyberg M, Nielsen S. Accurate PD-L1 protocols for non-small cell lung cancer can be developed for automated staining platforms with clone 22C3. Appl Immunohistochem Mol Morphol. 2017;25(6):381-5.

28. Gray RJ. A class of $\$ K \$$-sample tests for comparing the cumulative incidence of a competing risk. Ann Stat. 1988;16(3):1141-54. 
29. Fine JP, Gray RJ. A proportional hazards model for the subdistribution of a competing risk. J Am Stat Assoc. 1999;94(446):496-509.

30. Tuncel T, Karagoz B, Haholu A, Ozgun A, Emirzeoglu L, Bilgi O, et al. Immunoregulatory function of HLA-G in gastric cancer. Asian Pac J Cancer Prev. 2013;14(12):7681-4.

31. de Kruijf EM, Sajet A, van Nes JG, Natanov R, Putter H, Smit VT, et al. HLA-E and HLA-G expression in classical HLA class I-negative tumors is of prognostic value for clinical outcome of early breast cancer patients. J Immunol. 2010;185(12):7452-9.

32. Yie SM, Yang H, Ye SR, Li K, Dong DD, Lin XM. Expression of human leucocyte antigen $\mathrm{G}(\mathrm{HLA}-\mathrm{G})$ is associated with prognosis in non-small cell lung cancer. Lung Cancer. 2007;58(2):267-74.

33. Melsted WN, Johansen LL, Lock-Andersen J, Behrendt N, Eriksen JO, Bzorek M, et al. HLA class la and Ib molecules and FOXP3+ TILs in relation to the prognosis of malignant melanoma patients. Clin Immunol. 2017;183:191-7.

34. Ishitani A, Sageshima N, Lee N, Dorofeeva N, Hatake K, Marquardt H, et al. Protein expression and peptide binding suggest unique and interacting functional roles for $\mathrm{HLA}-\mathrm{E}, \mathrm{F}$, and $\mathrm{G}$ in maternal-placental immune recognition. J Immunol. 2003;171(3):1376-84.

35. Hviid TV. HLA-G in human reproduction: aspects of genetics, function and pregnancy complications. Hum Reprod Update. 2006;12(3):209-32.

36. Zeestraten EC, Reimers MS, Saadatmand S, Goossens-Beumer IJ, Dekker JW, Liefers GJ, et al. Combined analysis of HLA class I, HLA-E and $\mathrm{HLA}-\mathrm{G}$ predicts prognosis in colon cancer patients. Br J Cancer. 2014;110(2):459-68.

37. Lin A, Zhang X, Zhang RL, Zhang JG, Zhou WJ, Yan WH. Clinical significance of potential unidentified HLA-G isoforms without a1 domain but containing intron 4 in colorectal cancer patients. Front Oncol. 2018:8:361.

38. Zhang X, Lin A, Han QY, Zhang JG, Chen QY, Ye YH, et al. Intratumor heterogeneity of HLA-G expression in cancer lesions. Front Immunol. 2020;11:565759.

39. van de Water RB, Krijgsman D, Houvast RD, Vahrmeijer AL, PJK K. A critical assessment of the association between HLA-G expression by carcinomas and clinical outcome. Int J Mol Sci. 2021;22(15):8265.

40. Tronik-Le Roux D, Renard J, Vérine J, Renault V, Tubacher E, LeMaoult J, et al. Novel landscape of HLA-G isoforms expressed in clear cell renal cell carcinoma patients. Mol Oncol. 2017:11(11):1561-78.

41. Dapson RW. Macromolecular changes caused by formalin fixation and antigen retrieval. Biotech Histochem. 2007:82(3):133-40.

42. JVD A, Dessens CE, van de Water R, Houvast RD, PJK K, Krijgsman D. The molecular and functional characteristics of HLA-G and the interaction with its receptors: where to intervene for cancer immunotherapy? Int J Mol Sci. 2020;21:8678

43. Anna F, Bole-Richard E, LeMaoult J, Escande M, Lecomte M, Certoux JM, et al. First immunotherapeutic CAR-T cells against the immune checkpoint protein HLA-G. J Immunother Cancer. 2021;9(3):e001998.

44. Dolgin E. Gilead buys into Tizona's anti-HLA-G strategy. Cancer Discov. 2020;10(10):1433.

45. Wei XL, Wu QN, Chen DL, Zeng ZL, Lu JB, Liu ZX, et al. The clinical and biomarker association of programmed death ligand 1 and its spatial heterogeneous expression in colorectal cancer. J Cancer. 2018;9(23):4325-33.

46. Hecht M, Büttner-Herold M, Erlenbach-Wünsch $\mathrm{K}$, Haderlein M, Croner R, Grützmann $\mathrm{R}$, et al. PD-L1 is upregulated by radiochemotherapy in rectal adenocarcinoma patients and associated with a favourable prognosis. Eur J Cancer. 2016;65:52-60

47. Miller TJ, McCoy MJ, Hemmings C, Bulsara MK, lacopetta B, Platell CF. The prognostic value of cancer stem-like cell markers SOX2 and CD133 in stage III colon cancer is modified by expression of the immune-related markers FoxP3, PD-L1 and CD3. Pathology. 2017;49(7):721-30.

48. Alexander PG, McMillan DC, Park JH. A meta-analysis of CD274 (PD-L1) assessment and prognosis in colorectal cancer and its role in predicting response to anti-PD-1 therapy. Crit Rev Oncol Hematol. 2021;157:103147.

49. Werling RW, Yaziji H, Bacchi CE, Gown AM. CDX2, a highly sensitive and specific marker of adenocarcinomas of intestinal origin: an immunohistochemical survey of 476 primary and metastatic carcinomas. Am J Surg Pathol. 2003;27(3):303-10.

50. Aasebø K, Dragomir A, Sundström M, Mezheyeuski A, Edqvist PH, Eide GE, et al. CDX2: a prognostic marker in metastatic colorectal cancer defining a better BRAF mutated and a worse KRAS mutated subgroup. Front Oncol. 2020;10:8.
51. Slik K, Turkki R, Carpén O, Kurki S, Korkeila E, Sundström J, et al. CDX2 loss with microsatellite stable phenotype predicts poor clinical outcome in stage II colorectal carcinoma. Am J Surg Pathol. 2019;43(11):1473-82.

52. Galon J, Costes A, Sanchez-Cabo F, Kirilovsky A, Mlecnik B, Lagorce-Pagès C, et al. Type, density, and location of immune cells within human colorectal tumors predict clinical outcome. Science. 2006;313(5795):1960-4.

53. Wirta EV, Seppälä T, Friman M, Väyrynen J, Ahtiainen M, Kautiainen H, et al Immunoscore in mismatch repair-proficient and-deficient colon cancer. J Pathol Clin Res. 2017;3(3):203-13.

54. Bertelsen CA, Neuenschwander AU, Jansen JE, Wilhelmsen M, KirkegaardKlitbo A, Tenma JR, et al. Disease-free survival after complete mesocolic excision compared with conventional colon cancer surgery: a retrospective, population-based study. Lancet Oncol. 2015;16(22):161-8.

55. Schuster NA, Hoogendijk EO, Kok AAL, Twisk JWR, Heymans MW. Ignoring competing events in the analysis of survival data may lead to biased results: a nonmathematical illustration of competing risk analysis. J Clin Epidemiol. 2020;122:42-8.

\section{Publisher's Note}

Springer Nature remains neutral with regard to jurisdictional claims in published maps and institutional affiliations.
Ready to submit your research? Choose BMC and benefit from:

- fast, convenient online submission

- thorough peer review by experienced researchers in your field

- rapid publication on acceptance

- support for research data, including large and complex data types

- gold Open Access which fosters wider collaboration and increased citations

- maximum visibility for your research: over $100 \mathrm{M}$ website views per year

At BMC, research is always in progress.

Learn more biomedcentral.com/submissions 